University of Wollongong

Research Online

Australian Institute for Innovative Materials -

Papers

Australian Institute for Innovative Materials

$1-1-2016$

Integrated carbon/red phosphorus/graphene aerogel 3D architecture via advanced vapor-redistribution for high-energy sodium-ion batteries

Hong Gao

University of Wollongong, hg173@uowmail.edu.au

Tengfei Zhou

University of Wollongong, tz765@uowmail.edu.au

Yang Zheng

University of Wollongong, yz966@uowmail.edu.au

Yuqing Liu

University of Wollongong, yl037@uowmail.edu.au

Jun Chen

University of Wollongong, junc@uow.edu.au

See next page for additional authors

Follow this and additional works at: https://ro.uow.edu.au/aiimpapers

Part of the Engineering Commons, and the Physical Sciences and Mathematics Commons

Research Online is the open access institutional repository for the University of Wollongong. For further information contact the UOW Library: research-pubs@uow.edu.au 


\title{
Integrated carbon/red phosphorus/graphene aerogel 3D architecture via advanced vapor-redistribution for high-energy sodium-ion batteries
}

\author{
Abstract \\ Sodium ion batteries (SIBs), a potential alternative to lithium ion batteries (LIBs), have attracted \\ remarkable attention recently due to the abundant natural resources for their precursors and their low \\ cost.[1-3] The requirement of feasible electrode materials with high sodium storage capacity and good \\ cycling stability has promoted the exploration of various electrode materials for SIBs.
}

\section{Keywords}

phosphorus/graphene, via, aerogel, carbon/red, 3d, integrated, architecture, advanced, batteries, sodiumion, high-energy, vapor-redistribution

\author{
Disciplines \\ Engineering | Physical Sciences and Mathematics
}

\section{Publication Details}

Gao, H., Zhou, T., Zheng, Y., Liu, Y., Chen, J., Liu, H. \& Guo, Z. (2016). Integrated carbon/red phosphorus/ graphene aerogel 3D architecture via advanced vapor-redistribution for high-energy sodium-ion batteries. Advanced Energy Materials, 6 (21), 1601037-1-1601037-7.

\section{Authors}

Hong Gao, Tengfei Zhou, Yang Zheng, Yuqing Liu, Jun Chen, Hua-Kun Liu, and Zaiping Guo 
Article type: Full Paper

Integrated Carbon/red Phosphorus/graphene Aerogel Three-dimensional Architecture via Advanced Vapor-redistribution for High Energy Sodium-ion Batteries

Hong Gao ${ }^{1}$, Tengfei Zhou ${ }^{1}$, Yang Zheng ${ }^{1}$, Yuqing Liu ${ }^{2}$, Jun Chen ${ }^{2}{ }^{*}$, Huakun Liu ${ }^{\text {, }}$ Zaiping Guo ${ }^{1 *}$

H. Gao, T. Zhou, Y. Zheng, Prof. H. Liu, Prof. Z. Guo

Institute for Superconducting and Electronic Materials, School of Mechanical, Materials and Mechatronics Engineering,

University of Wollongong,

North Wollongong, NSW 2500, Australia

E-mail: zguo@uow.edu.au

Y. Liu, Prof. J. Chen

ARC Centre of Excellence for Electromaterials Science, Intelligent Polymer Research Institute, Australian Institute of Innovative Materials,

University of Wollongong,

North Wollongong, NSW 2522, Australia

E-mail: junc@uow.edu.au

Keywords: Red Phosphorus, Aerogel, Redistribution, Stability, Sodium-ion battery 


\begin{abstract}
Red phosphorus (P) is exceptionally useful as an electrode material for lithium-ion batteries and sodium-ion batteries, because it has $\sim 10$ times the theoretical capacity of its carbon based counterpart, but it remains a great challenge to rationally design P-based materials with stable structural reservoirs to counteract the large volume expansion, structural degradation and low conductivity problems during cycling. Herein, we have designed and fabricateed a threedimensional (3D) hierarchically integrated carbon/red phosphorus/graphene aerogel composite (C@P/GA) via an advanced vapor-redistribution technique. The as-prepared C@P/GA features red P nanoparticles 10-20 nm in size that are uniformly distributed and firmly sealed between the graphene aerogel and the carbon surface coating layer, which significantly improves the structural stability of the composite material and helps in maintaining the integrity of the electrode during electrochemical cycling. The 3D porous GA and carbon coating in the $\mathrm{C} @ \mathrm{P} / \mathrm{GA}$ composite provide an effective interconnected conductive network, as well as ideal elastic buffer interspaces to accommodate the volume expansion of red phosphorous, ensuring excellent electrochemical performance. The as-prepared C@P/GA electrode delivers a capacity of $1867 \mathrm{mAh} \cdot \mathrm{g}^{-1}$ after 100 cycles at $0.1 \mathrm{C}$. Moreover, a discharge capacity of $1095.5 \mathrm{mAh} \cdot \mathrm{g}^{-1}$ can be achived even after 200 cycles at $1 \mathrm{C}$.
\end{abstract}




\section{Introduction}

Sodium ion batteries (SIBs), a potential alternative to lithium ion batteries (LIBs), have attracted remarkable attention recently due to the abundant natural resources for their precursors and their low cost. ${ }^{[1-3]}$ The requirement of feasible electrode materials with high sodium storage capacity and good cycling stability has promoted the exploration of various electrode materials for SIBs.

Among all the anode material candidates, red phosphorus $(\mathrm{P})$ is regarded as an especially promising anode material for SIBs, because it is commercially available and has a high theoretical specific capacity $\left(2596 \mathrm{~mA} \mathrm{~h} \cdot \mathrm{g}^{-1}\right) .{ }^{[4-6]}$ Nevertheless, red phosphorus faces the challenges of low conductivity $\left(\sim 10^{-14} \mathrm{~S} / \mathrm{cm}\right)$ and large volumetric change $(\sim 400 \%)$ during the cycling process, leading to sluggish kinetics of electrochemical reactions and exceptional capacity fading. Several strategies have been proposed to conquer these impediments. ${ }^{[7-10]}$ For instance, it has been reported that the $\mathrm{P} /$ multi-walled carbon nanotube composite fabricated by a simple hand-grinding method could improve the electrochemical performance of $\mathrm{P}$ anode. ${ }^{[7 \mathrm{c}]}$ The P/graphite composites has also been prepared via high energy ball-milling method which stabilized the $\mathrm{P}$ electrode during electrochemical cycling. ${ }^{[8 \mathrm{c}]}$ Red P/single walled carbon nanotube and red P/CMK-3 composites with significant cycling stability improvement were synthesized via a vaporization-condensation process. ${ }^{[8 \mathrm{e}-\mathrm{f}]}$ In spite of some encouraging advancement, there are still severe issues hinder the electrochemical performance of P-based materials. For the most part, uneven particle size, limited coverage and poor electrical contact ascribed to the simply mechanical mixing of phosphorus and carbon matrixes result in the rapid capacity decay. The uncontrollability of red $\mathrm{P}$ distribution in the carbon matrix for traditional vaporization-condensation technique limits the electrodes electrochemical performance. Therefore, developing a novel P-based hybrid nanostructure by coupling the integrated carbon layer so as to enhance the conductivity and maintain the 
intimate contact between phosphorus and the conducting matrix, minimizing the particle size to control the phosphorus volume change and shorten the ion diffusion path together with highly conductive networks might be a promising approach to further improve the electrochemical performance.

Graphene aerogel (GA), a novel type of three-dimensional (3D) porous graphene architecture, has a high surface area and ample active sites, and the interconnected 3D porous structure is regarded as an ideal electrode structure for both electron and ion transfer during cycling. ${ }^{[11-13]}$ Herein, we fabricated a 3D integrated carbon/red phosphorus/graphene aerogel composite (C@P/GA) via an advanced vapor-redistribution strategy to achieve a uniform distribution of phosphorus nanoparticles (NPs) within the 3D graphene-based architecture. The vapor redistribution strategy that we developed includes two steps: (i) phosphorus micro particles (MPs) incorporation and (ii) localized phosphorus (NPs) vapor-redistribution processes. In contrast to the traditional vaporization-condensation strategy, firstly, the red $\mathrm{P}$ MPs were incorporated into the graphene hydrogel via an in-situ self-assembly approach, followed by vapor-phase polymerization of polypyrrole (PPy) in order to encapsulate the red P MPs into structure with well-defined porosity. The coating/deposition of PPy film is critical for maintaining the red $\mathrm{P}$ loading level during the following high-temperature vaporredistribution process. Lastly, a localized vapor-redistribution process was introduced to obtain uniformly deposited red P NPs, resulting in the C@P/GA.

The as-prepared C@P/GA composite features red P NPs uniformly distributed in the carbon covered GA matrix (C@GA), which integrated into 3D porous structure. The unique construction combines the following merits: firstly, the red P NPs are encapsulated in the matrix, which can fully accommodate the volume expansion of red $\mathrm{P}$ during cycling. Secondly, the integrated 3D conductive network could efficiently facilitate electron transfer and enable the conductive framework to be sufficiently immersed in the electrolyte. To the best of our knowledge, this is the first report on the synthesis of an integrated 3D porous 
C@P/GA composite with P NPs (10-20 nm) that are sealed in the C@GA matrix. In this way, the $\mathrm{P}$ content in the composite can be effectively maintained during the following vaporredistribution process, and due to the designed unique structure, P NPs were uniformly distributed and sealed in the C@GA matrix, so that the mobility of P particles on matrix was reduced, which greatly prevents the aggregation of $\mathrm{P}$ particles during electrochemical cycling when it is used as an anode material for SIBs The as-prepared C@P/GA electrode delivers a capacity of $\sim 1867 \mathrm{mAh} \cdot \mathrm{g}^{-1}\left(\sim 881 \mathrm{mAh} \cdot \mathrm{g}^{-1}\right.$ based on the composite) after 100 cycles at $0.1 \mathrm{C}$. At $1 \mathrm{C}$ it still delivers a capacity of $\sim 1095.5 \mathrm{mAh} \cdot \mathrm{g}^{-1}\left(\sim 517.1 \mathrm{mAh} \cdot \mathrm{g}^{-1}\right.$ based on the composite), even after 200 cycles. These integrated 3D porous C@ P/GA electrodes with high reversible capacity, stable cycling performance, and high rate capability, fabricated via the advanced vapor-redistribution technique could be promising anodes for SIBs.

\section{Results and Discussion}

Scheme 1 shows the synthesis process for the C@P/GA composite. The preparation process includs three steps: ( i ) An in-situ self-assembly process to obtain the 3D porous graphene-hydrogel-encapsulated red $\mathrm{P}$ precursor. The precursors with different amount of $\mathrm{P}$ (from $30 \%$ to $90 \%$ ) were also prepared. (ii) Vapor phase deposition of polypyrrole on the precursor in a chamber. The vapor phase deposition process is intended to produce conducting polymer layers on certain substrates with high conductivity and uniform distribution. ${ }^{[14]}$ (iii) Vapor-redistribution of red $\mathrm{P}$ by annealing the precursor in pure argon atmosphere to obtain the C@P/GA composite. For comparison, composites obtained from step (i) (pre-P/GA with red P MPs), and from steps (i) and (iii) (P/GA with red P NPs and uniform P distribution, but without carbon coating) were also prepared. Please note that the vapor-redistribution process was conducted in a sealed tube at $400{ }^{\circ} \mathrm{C}$ for just $20 \mathrm{~min}$, followed by a quench process. In addition, the PPy would be carbonized at the same time. The carbon coverage, short 
redistribution time, and quench process would be beneficial for maintaining the loading level of red $\mathrm{P}$ in the matrix.

Figure 1a presents the X-ray diffraction (XRD) patterns of commercial red P, GA, preP/GA, P/GA, and C@P/GA composites. The XRD pattern of the commercial red P with the first sharp peak centered at $\sim 15^{\circ}$ is similar to that of red P reported previously, ${ }^{[15]}$ a hump at $22^{\circ} \sim 28^{\circ}$ appeared in the XRD pattern of GA. The pre-P/GA pattern shows the distinctive XRD peaks of GA and red $\mathrm{P}$, and indicates that the interlayer spacing of graphene is $0.384 \mathrm{~nm}$, while the interlayer spacing of graphene in P/GA and C@P/GA was calculated to be 0.358 $\mathrm{nm}$ and $0.369 \mathrm{~nm}$, respectively. Compared with pre-P/GA, the decreased interlayer spacing of graphene in $\mathrm{P} / \mathrm{GA}$ and $\mathrm{C} @ \mathrm{P} / \mathrm{GA}$ suggests the removal of functional groups during the redistribution process. ${ }^{[16]}$ The interlayer spacing of graphene in $\mathrm{C} @ \mathrm{P} / \mathrm{GA}$ is increased compared to that in P/GA, due to the decomposition of PPy during the vapor-redistribution process, which releases gas and separates the adjacent graphene sheets. ${ }^{[17]}$ Both P/GA and $\mathrm{C} @ \mathrm{P} / \mathrm{GA}$ retain the distinctive XRD peaks of GA and red $\mathrm{P}$, and the lower peak intensity of phosphorus demonstrating the coverage of the GA and C@GA, respectively. ${ }^{[10 \mathrm{~d}, 18]}$ Besides, the existence of the peaks relate to $\mathrm{P}$, implying some $\mathrm{P}$ particles are not covered by the matrix. Figure 1b shows Raman spectra of the commercial red P, GA, and pre-P/GA, P/GA, and C@P/GA composites. The commercial red P shows three major peaks between 300 and 500 $\mathrm{cm}^{-1[8 \mathrm{~h}]}$, and the GA pattern reveals the characteristic D band $\left(1325 \mathrm{~cm}^{-1}\right)$ and $\mathrm{G}$ band $(1590$ $\left.\mathrm{cm}^{-1}\right){ }^{[17]}$ The pre-P/GA, P/GA, and C@P/GA composites clearly combine the characteristic peaks of red $\mathrm{P}$ and GA, while the peak intensity related to the phosphorus in P/GA and C@P/GA composites are largely decrease, which is in agreement with the XRD results. Figure S1 in the Supporting Information presents the Raman spectra of PPy/P/GA (precursor of C@P/GA without the vapor-redistribution process) compared with pure PPy, GA, and red P. The presence of a characteristic peak of PPy in PPy/P/GA suggests successful coverage of with PPy. Figure 1c exhibits a digital photograph of the as-prepared C@P/GA composite. 
The morphology of the pre-P/GA, P/GA, and C@P/GA composites was investigated via field emission scanning electron microscopy (SEM) (Figure 1d-f). Figure 1d exhibits the relatively large $\mathrm{P}$ particles incorporated in the graphene layers for the pre-P/GA composite, which is consistent with the corresponding transmission electron microscope (TEM) images and energy dispersive X-ray spectroscopy (EDS) element mapping analysis (Figure S2). The particle size of red $\mathrm{P}$ in P/GA composite is decreased sharply, which is attributed to the vaporredistribution process (Figure 1e). The corresponding TEM and EDS element mapping analysis of P/GA further demonstrates the homogenous distribution of red P NPs (Figure S3). For C@P/GA (Figure 1f), the pore size is much smaller and the sheets are more wrinkled than that of the pre-P/GA and P/GA composites, which is possibly due to the shrinkage of the gel during the vapor phase deposition process for the coverage with PPy. ${ }^{[14 c]}$ The crumpled matrix could further activate ion kinetics and accommodate the volume changes of red $\mathrm{P}$ during cycling. The sheets of the composite obviously lose transparency and even become thicker, due to the carbon coverage. In addition, the high magnificent SEM images of C@P/GA are shown in Figure S4, which demonstrates the P particles (10-20nm) sealed in the crumpled C@GA matrix. The thermogravimetric analysis (TGA) curves (Figure S5) show that the content of red $\mathrm{P}$ in $\mathrm{C} @ \mathrm{P} / \mathrm{GA}$ is almost two times higher than that of the P/GA, suggesting that this uniquely designed structure is beneficial for maintaining the loading level of P during the vapor-redistribution process. The TGA of PPy (Figure S6) demonstrates that the PPy film started its carbonization from $\sim 250{ }^{\circ} \mathrm{C}$, which is much lower than the P vaporization temperature $\left(400{ }^{\circ} \mathrm{C}\right)$. This means the deposited PPy film carbonized first before the $\mathrm{P}$ vapour-redistribution, which could help to keep most $\mathrm{P}$ nanoparticles inside the porous structure rather than on the outer surface of the resultant $\mathrm{C} @ \mathrm{P} / \mathrm{GA}$. This kind of encapsulation of P NPs inside porous structures might be able to protect the P NPs from the direct exposure to the electrolyte, with the potential result of improved cycling stability during the following charge-discharge process. 
Figure 2a-g shows SEM images of pre-P/GA composites with the amounts of phosphorus varying from $30 \%$ to $90 \%$. The regular increase in the amount of phosphorus in the GA can be clearly observed, and the corresponding digital photograph demonstrate the collapse of graphene hydrogel when the amount of red $\mathrm{P}$ increases to $90 \%$. Considering the upper limit for loading of the graphene hydrogel, pre-P/GA with red P content of $80 \%$ was selected for further study. In order to confirm a suitable vapor-redistribution temperature, different heating temperatures from 200 to $500{ }^{\circ} \mathrm{C}$ were studied, and the regular changes in the red $\mathrm{P}$ particles were investigated via SEM (Figure S7). Considering the red $\mathrm{P}$ particle size and loading amount, the temperature of $400{ }^{\circ} \mathrm{C}$ was selected for the synthesis of C@P/GA composite. The SEM images of pure red $\mathrm{P}$ particles were also investigated for comparison purposes (Figure S8).

The morphology of C@P/GA composite was further investigated via transmission electron microscopy (TEM). The image exhibits red P NPs (with sizes in the range of 10-20 nm) uniformly encapsulated in the crumpled matrix (Figure 3a). The high-resolution TEM (HRTEM) image reveals a typical red P NP that is entirely covered by carbon and graphene sheets (Figure 3b). The corresponding STEM and EDS element mapping images of C@ P/GA composite suggest the presence of carbon, nitrogen, and phosphorus components, further demonstrating the homogeneous coverage by carbon and the uniform distribution of red $\mathrm{P}$ in the structure (Figure 3c-f and Figure S9). In addition, TEM images of pure GA and C@GA were also collected (Figure S10). The chemical composition was further investigated via Xray photoelectron spectroscopy (XPS). Figure 3g displays the XPS survey spectras for the pre-P/GA, P/GA and C@P/GA composites. Compared with pre-P/GA and P/GA composites, a N 1s peak centered at $\sim 400 \mathrm{eV}$ can be observed in the XPS spectrum of the C@P/GA composite, which shows that the content of $\mathrm{N}$ in the $\mathrm{C} @ \mathrm{P} / \mathrm{GA}$ composite is about 4.1 at.\%. The $\mathrm{N}$ element comes from the decomposition of PPy, the carbon source for the C@P/GA. Figure 3h exhibits the high-resolution XPS C 1s spectrum of C@P/GA, which could be fitted 
into several spectral peaks: a dominant peak for $s p^{2}-\mathrm{C}$ at $284.8 \mathrm{eV}$, along with other weaker bands, including $s p^{3}-\mathrm{C}$ at $285.5 \mathrm{eV}, \mathrm{N}-s p^{3} \mathrm{C}$ at $287.5 \mathrm{eV}$, and $\mathrm{C}-\mathrm{C}=\mathrm{O}$ at $289 \mathrm{eV}$, respectively. ${ }^{[14,19]}$ The presence of the $\mathrm{N}-s p^{3} \mathrm{C}$ spectral peak would be a sign of enhanced electrical conductivity and easy charge transfer during the electrochemical process. ${ }^{[20]}$

Figure 4 presents the electrochemical performances of the as-prepared pre-P/GA, P/GA, and C@P/GA composites. Unless otherwise noted, all specific capacities here are calculated based on the weight of phosphorus. Figure 4a shows cyclic voltammetry $(\mathrm{CV})$ curves of the initial five scans for $\mathrm{C} @ \mathrm{P} / \mathrm{GA}$ composite at a scan rate of $0.1 \mathrm{mV} \mathrm{s}^{-1}$ between 0.01 and $2 \mathrm{~V}$. Figure $4 \mathbf{b}$ presents the $1^{\text {st }}, 2^{\text {nd }}$ and $100^{\text {th }}$ charge/discharge curves of the C@P/GA electrode at $0.1 \mathrm{C}$ between 0 and $2 \mathrm{~V}$. In the $\mathrm{CV}$ curves, a broad peak at $\sim 0.8 \mathrm{~V}$ is detected during the first cathodic scan, due to the formation of the solid electrolyte interphase (SEI) layer, corresponding to a plateau at $\sim 0.8 \mathrm{~V}$ in the initial discharge curve in the charge/discharge profiles. The following two discharge curves are composed of a downward sloping region from the beginning to $\sim 0.5 \mathrm{~V}$, an inclined plateau from $\sim 0.5$ to $\sim 0.12 \mathrm{~V}$ (consistent with a hump around $0.35 \mathrm{~V}$ in the subsequent cathodic scans in the CV curves), and another sloping region from $\sim 0.12$ to $\sim 0.01 \mathrm{~V}$. The charge curves include an upward sloping region $(0.06$ to $\sim 0.4 \mathrm{~V}$ ), three slightly inclined plateaus: $\sim 0.4$ to $\sim 0.6 \mathrm{~V}, \sim 0.6$ to $\sim 0.75 \mathrm{~V}$, and $\sim 0.75$ to $\sim 0.9 \mathrm{~V}$ ( consistent with peaks at $\sim 0.62 \mathrm{~V}, \sim 0.73 \mathrm{~V}$, and $\sim 0.96 \mathrm{~V}$ in the $\mathrm{CV}$ anodic scans), with a following sloping region up to $2 \mathrm{~V}$. The cycling performances of the pre-P/GA, P/GA, C@P/GA, red P, and C@GA electrodes at $0.1 \mathrm{C}$ are presented in Figure 4c. Dramatic capacity fading can be clearly observed from the pure red $\mathrm{P}$ cycling curves, which has a discharge capacity of $993.4 \mathrm{mAh} \cdot \mathrm{g}^{-1}$ in the second cycle, and decreases to $181 \mathrm{mAh} \cdot \mathrm{g}^{-1}$ only after 50 cycles. Compared to red $\mathrm{P}$, the pre-P/GA electrode shows an increased second cycle discharge capacity of $2234.6 \mathrm{mAh} \cdot \mathrm{g}^{-1}$ with an initial coulombic efficiency of $70.5 \%$, although its cycling performance is still very poor, with a quick drop in discharge capacity of 507.3 $\mathrm{mAh} \cdot \mathrm{g}^{-1}$ after 100 cycles, and the average coulombic efficiency of pre-P/GA is $~-94.4 \%$ 
(Figure S11a). A relatively stable cycling performance is observed for P/GA electrode, in which the capacity gradually decreases from the second discharge capacity of 2041.8 to $1589.8 \mathrm{mAh} \cdot \mathrm{g}^{-1}$ after 100 cycles, and the initial and average coulombic efficiency of P/GA electrode are $63.5 \%$ and $\sim 95.9 \%$, respectively (Figure S11b). The C@P/GA electrode, however, shows more stable electrochemical performance than that of the P/GA electrode. The C@P/GA electrode delivers an initial coulombic efficiency of $75.4 \%$ and a second discharge capacity of $2085 \mathrm{mAh} \cdot \mathrm{g}^{-1}$. A high capacity of $1867 \mathrm{mAh} \cdot \mathrm{g}^{-1}$ is still obtained after 100 cycles with a capacity retention of $89.5 \%$ (relative to the second cycle) and an average coulombic efficiency of $\sim 97.3 \%$ (Figure S11c). The C@GA electrode behaves the second discharge capacity of $368.8 \mathrm{mAh} \cdot \mathrm{g}^{-1}$ and $212.3 \mathrm{mAh} \cdot \mathrm{g}^{-1}$ after 100 cycles. From the above results, we can conclude that the cycling performances of the P/GA and C@P/GA electrodes are better than that of the pure red $\mathrm{P}$ and pre-P/GA electrodes, suggesting that the smaller particle size and uniform distribution of red $\mathrm{P}$ are critical points for improving the cycling performance of the electrodes. In addition, the best electrochemical stability of the C@P/GA electrode demonstrates the significance of the coverage of the red P NPs.

Figure 4d shows the rate capability of the pre-P/GA, P/GA, and C@P/GA electrodes. Obviously, the C@P/GA electrode exhibits the best rate capability: it delivers discharge capacities of $2042.5,1908.6,1648.0$, and $1354.0 \mathrm{mAh} \cdot \mathrm{g}^{-1}$ at the current densities of $0.1 \mathrm{C}, 0.2$ C, $0.5 \mathrm{C}$ and $1 \mathrm{C}$ respectively. Even at the high current density of $2 \mathrm{C}$, a reversible capacity of $878.6 \mathrm{mAh} \cdot \mathrm{g}^{-1}$ can be obtained. When the current density is reversed back to $1 \mathrm{C}$ and $0.1 \mathrm{C}$, reversible capacity of 1230.2 and $1899.4 \mathrm{mAh} \cdot \mathrm{g}^{-1}$, respectively, still can be reached. At the relatively higher current density of $1 \mathrm{C}$, the $\mathrm{C} @ \mathrm{P} / \mathrm{GA}$ electrode still delivers a discharge capacity of $1095.5 \mathrm{mAh} \cdot \mathrm{g}^{-1}$, even after 200 cycles, with a capacity retention of $66.9 \%$ and an average coulombic efficiency of $\sim 93.3 \%$. (Figure 4e). The excellent electrochemical performance of C@P/GA electrode should be ascribed to the unique design of the structure, as shown in Scheme 2, which illustrates the electrochemical mechanisms of the C@P/GA 
electrode in the SIB system. During the electrochemical process $\left(\mathrm{P}+3 \mathrm{Na}^{+}+3 \mathrm{e}^{-} \leftrightarrow \mathrm{Na}\right.$ 至), the red P NPs are confined geometrically in the C@GA matrix, which increases the interface contact between the red $\mathrm{P}$ and the conductive network, and accommodates the red $\mathrm{P}$ volume expansion as well. In addition, the highly conductive 3D porous structure provides a rapid and efficient electron transport network to improve the conductivity, which was demonstrated by electrochemical impedance spectroscopy (Figure S12). The charge transfer resistance of C@P/GA electrode after 30 cycles is $177.2 \Omega$, lower than those of the P/GA and pre-P/GA electrodes, $363.6 \Omega$ and $434.3 \Omega$, respectively. What is more, the stability of the structure ensures an integrated, highly conductive 3D porous network, which provides rapid and efficient electron transport paths. Figure S13 presents SEM images of C@P/GA electrode before and after the charge-discharge process, which shows that the $3 \mathrm{D}$ porous structure is still maintained after 100 cycles, indicating the superior structural stability of the electrode.

\section{Conclusions}

In summary, a new advanced synthesis protocol has been developed to incorporate red P NPs into 3D porous $\mathrm{C} @ \mathrm{GA}$ matrix with a uniform distribution through a localized in-situ vaporredistribution strategy. The red $\mathrm{P}$ nanoparticles with $10-20 \mathrm{~nm}$ in size are uniformly distributed and firmly sealed in a well-defined 3D porous C@ GA framework, which not only provides a 3D conductive network for electron transfer, but also effectively accommodates/buffers the large volume changes of red $\mathrm{P}$ during the charge-discharge process, helping to maintain the integrity of the electrode and enhance the electrochemical performance. The as-prepared C@P/GA electrode delivers a high capacity of $1867 \mathrm{mAh} \cdot \mathrm{g}^{-1}$ after 100 cycles at $0.1 \mathrm{C}$ and demonstrates a significant reversible capacity of $1095.5 \mathrm{mAh} \cdot \mathrm{g}^{-1}$, even at $1 \mathrm{C}$ after 200 cycles. This evidence strongly confirms that C@P/GA would be a promising anode material for SIBs, and shows a demo that the synthesis protocols that we 
have developed could also be instructive for incorporating and encapsulating active materials with large-volume expansion and moderate evaporation temperatures, such as sulfur.

\section{Supporting Information}

\section{Acknowledgments}

Support from the Australian Research Council (ARC) through an ARC Discovery project (DP1094261) and Future Fellowship (FT150100109) are gratefully acknowledged. The authors would also like to thank the Australian National Fabrication Facility (ANFF) for equipment access, the Electron Microscopy Centre (EMC) at the University of Wollongong for the electron microscopy characterizations, and Dr. Tania Silver for critical reading of the manuscript and valuable remarks.

Received: ((will be filled in by the editorial staff))

Revised: ((will be filled in by the editorial staff)) Published online: ((will be filled in by the editorial staff))

[1] a) Y. Yan, Y. Yin, Y. Guo, L. Wan, Adv. Energy Mater. 2014, 4, 1301584; b) T. Zhou, W. Pang, C. Zhang, J. Yang, Z. Chen, H. Liu, Z. Guo, ACS Nano, 2014, 8, 8323; c) S. Wang, L. Xia, L. Yu, L. Zhang, H. Wang, X. Lou, Adv. Energy Mater. 2016, 6, 1502217; d) C. Zhu, P. Kopold, P. van Aken, J. Maier, Y. Yu, Adv. Mater. 2016, 28, 2409;

[2] a) Y. Wen, K. He, Y. Zhu, F. Han, Y. Xu, I. Matsuda, Y. Ishii, J. Cumings, C. Wang, Nat. Commun. 2014, 5, 4033; b) J. Xu, M. Wang, N. P. Wickramaratne, M. Jaroniec, S. Dou, L. Dai, Adv. Mater., 2015, 27, 2042; c) L. Qie, W. Chen, X. Xiong, C. Hu, F. Zou, P. Hu, Y. Huang, Adv. Sci. 2015, 2, 1500195.

[3] a) Y. X. Wang, J. Yang, S. L. Chou, H. K. Liu, W. X. Zhang, D. Zhao, S. X. Dou, Nat. Commun. 2015, 6, 8689; b) Z. Zeng, X. Jiang, R. Li, D. Yuan, X. Ai, H. Yang, Y. Cao, 
Adv. Sci. 2016, 1600066; c) Y. Zheng, T. Zhou, C Zhang, J. Mao, H. Liu, Z. Guo, Angew.Chem. Int. Ed. 2016, 55, 3408.

[4] a) T. R. Jow, L. W. Shacklette, M. Maxfield, D. Vernick, J. Electrochem. Soc. 1987, 134, 1730; b) Y. Ma, M. M. Doeff, S. J. Visco, L. C. De Jonghe, J. Electrochem. Soc. 1993, 140, 2726; c) L. D. Ellis, T. D. Hatchard, M. N. Obrovac, J. Electrochem. Soc. 2012, 159, A1801; d) S. Komaba, Y. Matsuura, T. Ishikawa, N. Yabuuchi, W. Murata, S. Kuze, Electrochem. Commun. 2012, 21, 65; e) T. Yamamoto, T. Nohira, R. Hagiwara, A. Fukunaga, S. Sakai, K. Nitta, S. Inazawa, J. Power Sources 2013, 237, 98.

[5] a) L. Li, K. H. Seng, D. Li, Y. Xia, H. K. Liu, Z. Guo, Nano Research, 2014, 7, 14661476; b) L. Hu, X. Zhu, Y. Du, Y. Li, X. Zhou, J. Bao, Chem. Mater. 2015, 27, 81388145.

[6] a) L. Wu, X. Hu, J. Qian, F. Pei, F. Wu, R. Mao, X. Ai, h. Yang, Y. Cao, Energy Environ. Sci. 2014, 7, 323; b) N. Wang, Z. Bai, Y. Qian, J. Yang, Adv. Mater. 2016, DOI: 10.1002/adma.201505918.

[7] a) P. R. Abel, Y.-M. Lin, T. de Souza, C.-Y. Chou, A. Gupta, J. B. Goodenough, G. S. Hwang, A. Heller, C. B. Mullins, J. Phys. Chem. C 2013, 117, 18885; b) L. Baggetto, J. K. Keum, J. F. Browning, G. M. Veith, Electrochem. Commun. 2013, 34, 41; c) W.-J. Li, S.-L. Chou, J.-Z. Wang, H.-K. Liu, S.-X. Dou, Nano Lett. 2013, 13, 5480.

[8] a) N. Yabuuchi, Y. Matsuura, T. Ishikawa, S. Kuze, J.-Y. Son, Y.-T. Cui, H. Oji, S. Komaba, ChemElectroChem 2014, 1, 580; b) Y. Kim, Y. Park, A. Choi, N.-S. Choi, J. Kim, J. Lee, J. H. Ryu, S. M. Oh, K. T. Lee, Adv. Mater. 2013, 25, 3045; c) J. Sun, G. Zheng, H.-W. Lee, N. Liu, H. Wang, H. Yao, W. Yang, Y. Cui, Nano Lett. 2014, 14, 4573; d) J. Qian, X. Wu, Y. Cao, X. Ai, H. Yang, Angew. Chem. 2013, 125, 4731; e) Y. Zhu, Y. Wen, X. Fan, T. Gao, F. Han, C. Luo, S.-C. Liou, C. Wang, ACS Nano. 2015, 9, 3254; f) W. Li, Z. Yang, M. Li, Y. Jiang, X. Wei, X. Zhong, L. Gu, Y. Yu, Nano Lett. 2016, 16, 1546; g) J. Sun, H.-W. Lee, M. Pasta, H. Yuan, G. Zheng, Y. Sun, Y. Li, Y. 
Cui, Nat. Nanotechnol. 2015, 10, 980; h) J. Qian, D. Qiao, X. Ai, Y. Cao, H. Yang, Chem. Commun. 2012, 48, 893; i) L. Pei, Q. Zhao, C. Chen, J. Liang, J. Chen, ChemElectroChem 2015, 2, 1652.

[9] a) C.-M. Park, J.-H. Kim, H. Kim, H.-J. Sohn, Chem. Soc. Rev. 2010, 39, 3115; b) W.-J. Zhang, J. Power Sources 2011, 196, 877; c) R. Yi, F. Dai, M. L. Gordin, S. Chen, D. Wang, Adv. Energy. Mater. 2013, 3, 295; d) J. Song, S. Chen, M. Zhou, T. Xu, D. Lv, M. L. Gordin, T. Long, M. Melnyk, D. Wang, J. Mater. Chem. A 2014, 2, 1257.

[10] a) J. Song, Z. Yu, M. L. Gordin, S. Hu, R. Yi, D. Tang, T. Walter, M. Regula, D. Choi, X. Li, A. Manivannan, D. Wang, Nano Lett. 2014, 14, 6329; b) Z. Yu, J. Song, M. L. Gordin, R. Yi, D. Tang, D. Wang, Adv. Sci. 2015, 2, 1400020; c) C. Marino, A. Debenedetti, B. Fraisse, F. Favier, L. Monconduit, Electrochem. Commun. 2011, 13, 346; d) L. Wang, X. He, J. Li, W. Sun, J. Gao, J. Guo, C. Jiang, Angew. Chem. Int. Ed. 2012, 51, 9034 .

[11] a) W. Wei, S. Yang, H. Zhou, I. Lieberwirth, X. Feng, K. Müllen, Adv. Mater. 2013, 25, 2909; b) Z.-S. Wu, A. Winter, L. Chen, Y. Sun, A. Turchanin, X. Feng, K. Müllen, Adv. Mater. 2012, 24, 5130; c) Z.-S. Wu, S. Yang, Y. Sun, K. Parvez, X. Feng, K. Müllen, J. Am. Chem. Soc. 2012, 134, 9082.

[12] M. A. Worsley, P. J. Pauzauskie, T. Y. Olson, J. Biener, J. H. Satcher, T. F. Baumann, J. Am. Chem. Soc. 2010, 132, 14067.

[13] a) J. Yuan, J. Zhu, H. Bi, X. Meng, S. Liang, L. Zhang, X. Wang, Phys. Chem. Chem. Phys. 2013, 15, 12940; b) Y. Huang, D. Wu, S. Han, S. Li, L. Xiao, F. Zhang, X. Feng, ChemSusChem 2013, 6, 1510.

[14] M. Wang, J. Wang, Y. Hou, D. Shi, D. Wexler, S. D. Poynton, R. C. T. Slade, W. Zhang, H. Liu, J. Chen, ACS Appl. Mater. Interface. 2015, 7, 7066.

[15] J. M. Zaug, A. K. Soper, S. M. Clark, Nat. Mater. 2008, 7, 890. 
[16] Z.-Y. Sui, Q.-H. Meng, J.-T. Li, J.-H. Zhu, Y. Cui, B.-H. Han, J. Mater. Chem. A 2014, 2, 9891.

[17] Z.-Y. Sui, C. Wang, Q.-S. Yang, K. Shu, Y.-W. Liu, B.-H. Han, G. G. Wallace, J. Mater. Chem. A 2015, 3, 18229.

[18] C. Marino, L. Boulet, P. Gaveau, B. Fraisse, L. Monconduit, J. Mater. Chem. 2012, 22, 22713.

[19] a) J. Xu, M. Wang, N. P. Wickramaratne, M. Jaroniec, S. Dou, L. Dai, Adv. Mater. 2015, 27, 2042; b) C.-C. Teng, C.-C. M. Ma, C.-H. Lu, S.-Y. Yang, S.-H. Lee, M.-C. Hsiao, M.-Y. Yen, K.-C. Chiou, T.-M. Lee, Carbon 2011, 49, 5107.c) D. Wei, Y. Liu, Y. Wang, H. Zhang, L. Huang, G. Yu, Nano Lett. 2009, 9, 1752.

[20] J. P. Paraknowitsch, A. Thomas, Energy. Environ. Sci. 2013, 6, 2839. 


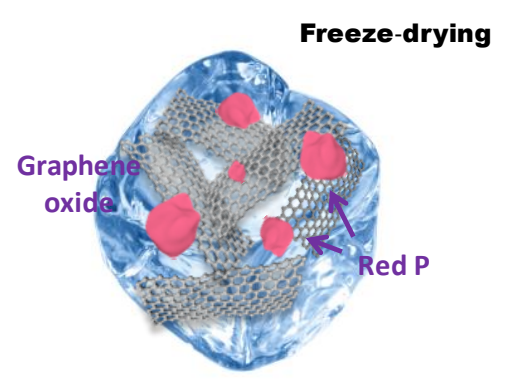

$$
\text { i In-situ }
$$

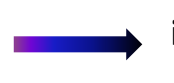

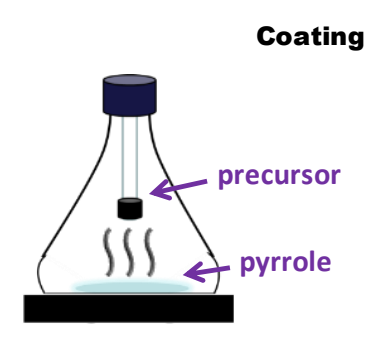

ii Vapour phase polymerization

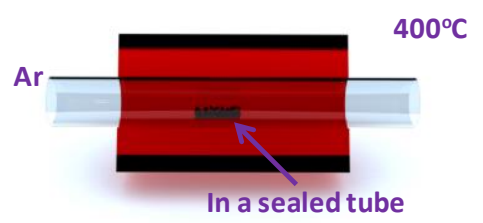

iii Vapour-redistribution

process
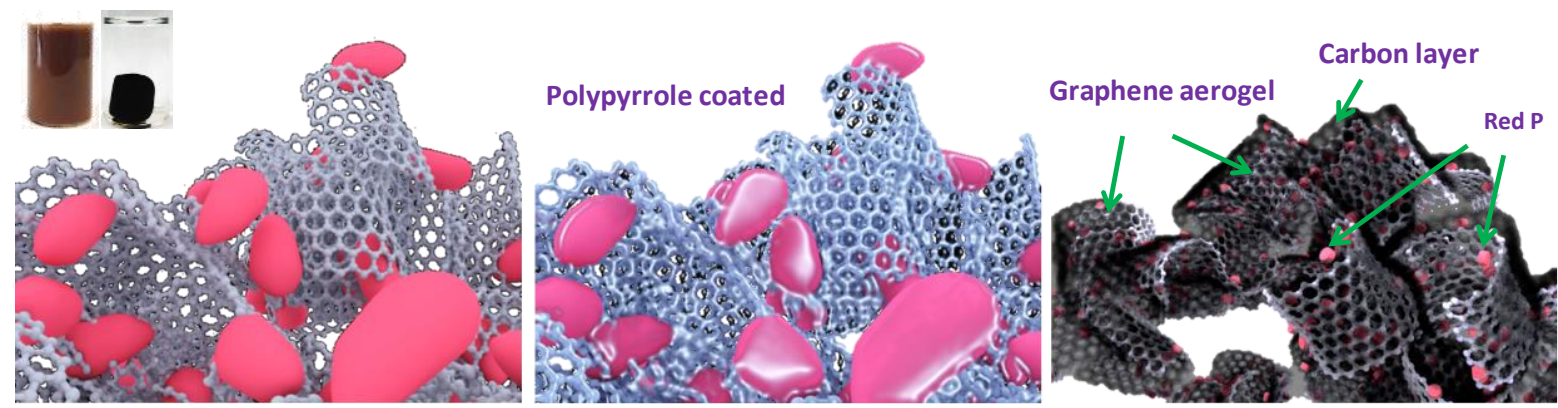

Scheme 1. Schematic illustration of the synthesis process for C@P/GA composite in three steps. ( i ) An in-situ self-assembly process to obtain the 3D porous graphene-hydrogelencapsulated red $\mathrm{P}$ precursor. (ii) Vapor phase deposition of polypyrrole on the precursor in a chamber. (iii) Vapor-redistribution of red $\mathrm{P}$ by annealing the precursor in pure argon atmosphere to obtain the C@P/GA composite. 

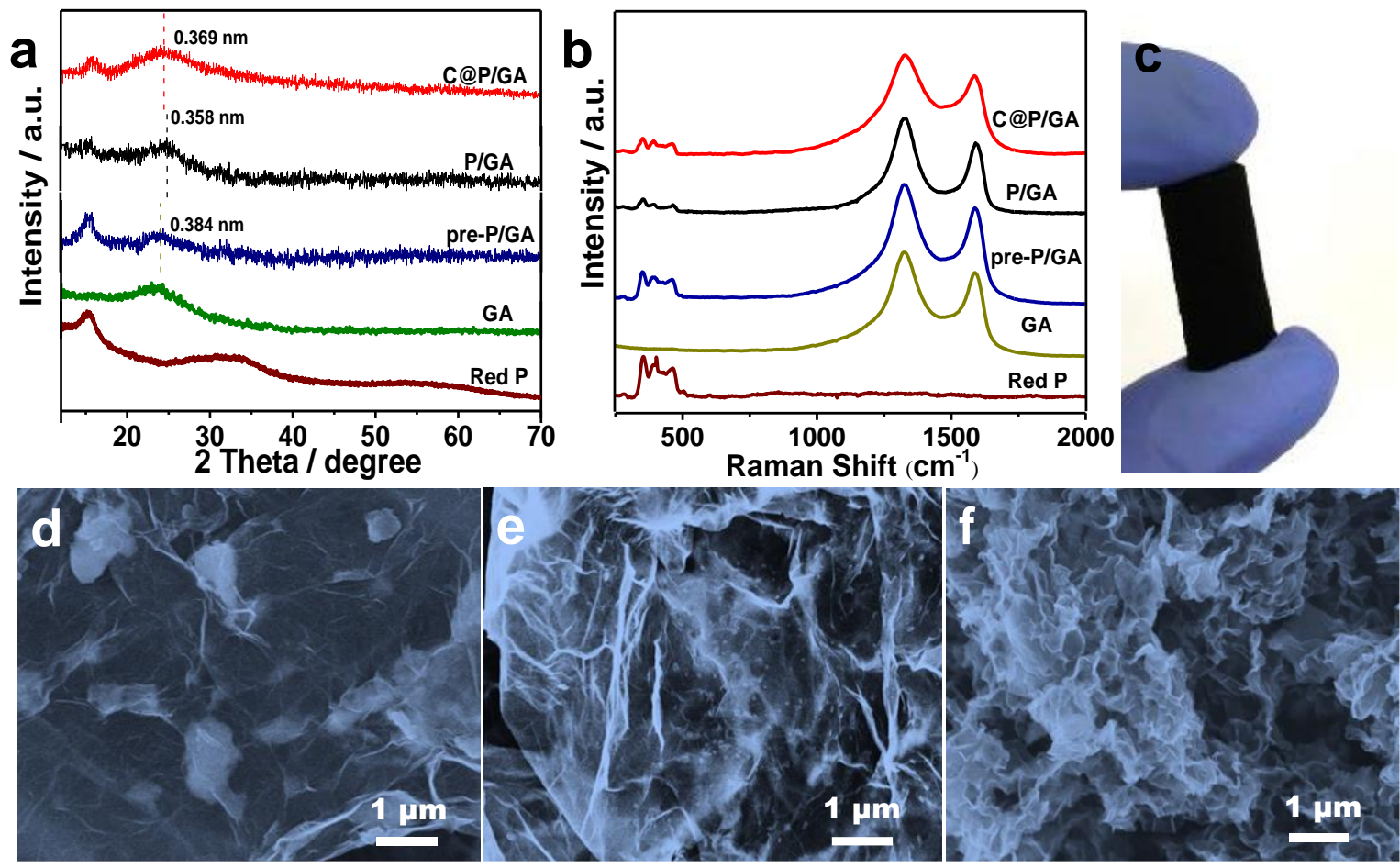

Figure 1. a) XRD patterns and (b) Raman spectra of red $\mathrm{P}, \mathrm{GA}$, pre-P/GA, P/GA, and C@P/GA composites. c) Digital photograph of the as-prepared C@P/GA composite. (d-f) SEM images of d) pre-P/GA, e) P/GA, and f) C@P/GA. 

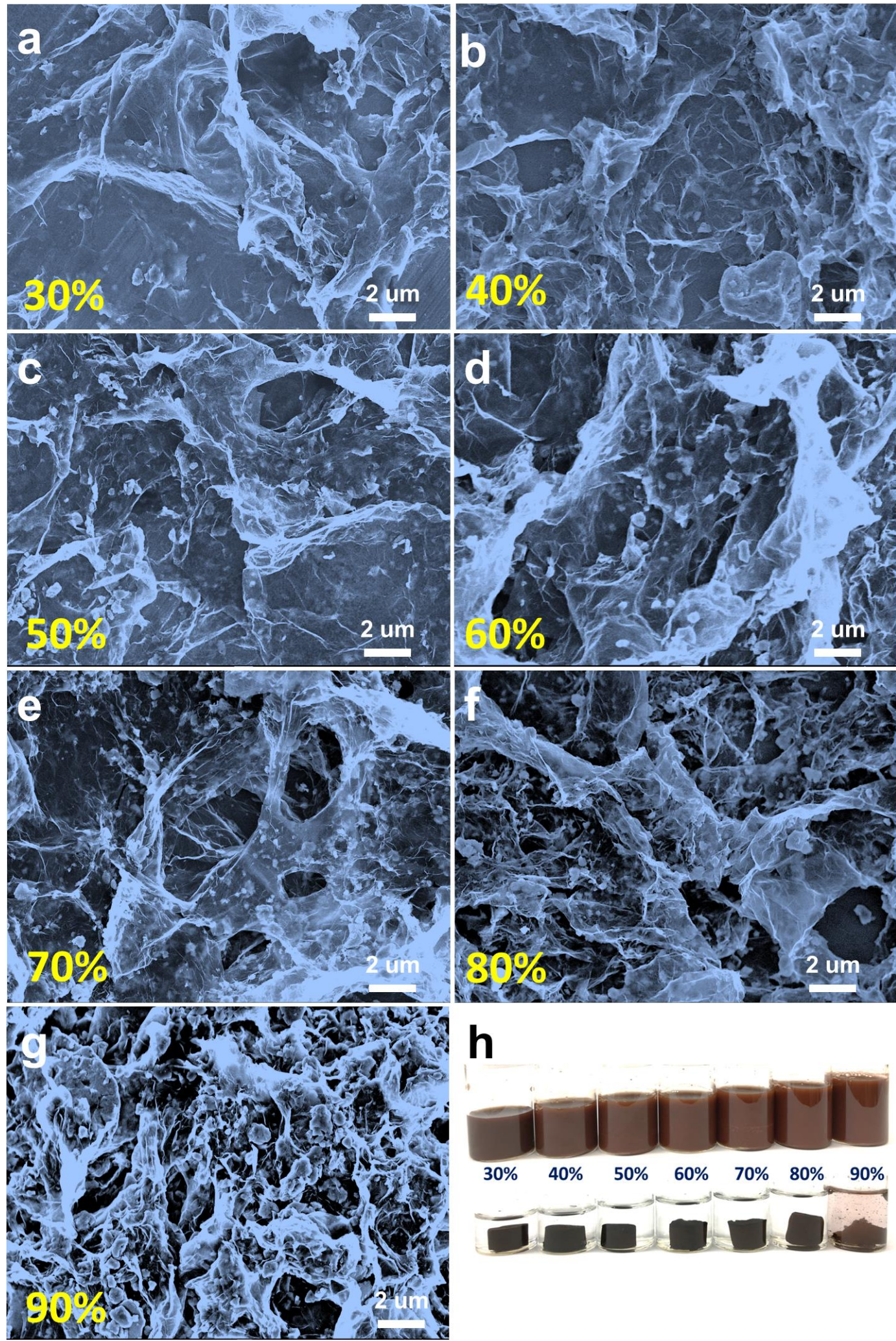

Figure 2. a-g) SEM images of pre-P/GA composites with different amounts of red $\mathrm{P}$, and h) corresponding digital photograph of the precursor solutions and pre-P/GA composites with different concentrations of $\mathrm{P}$. 

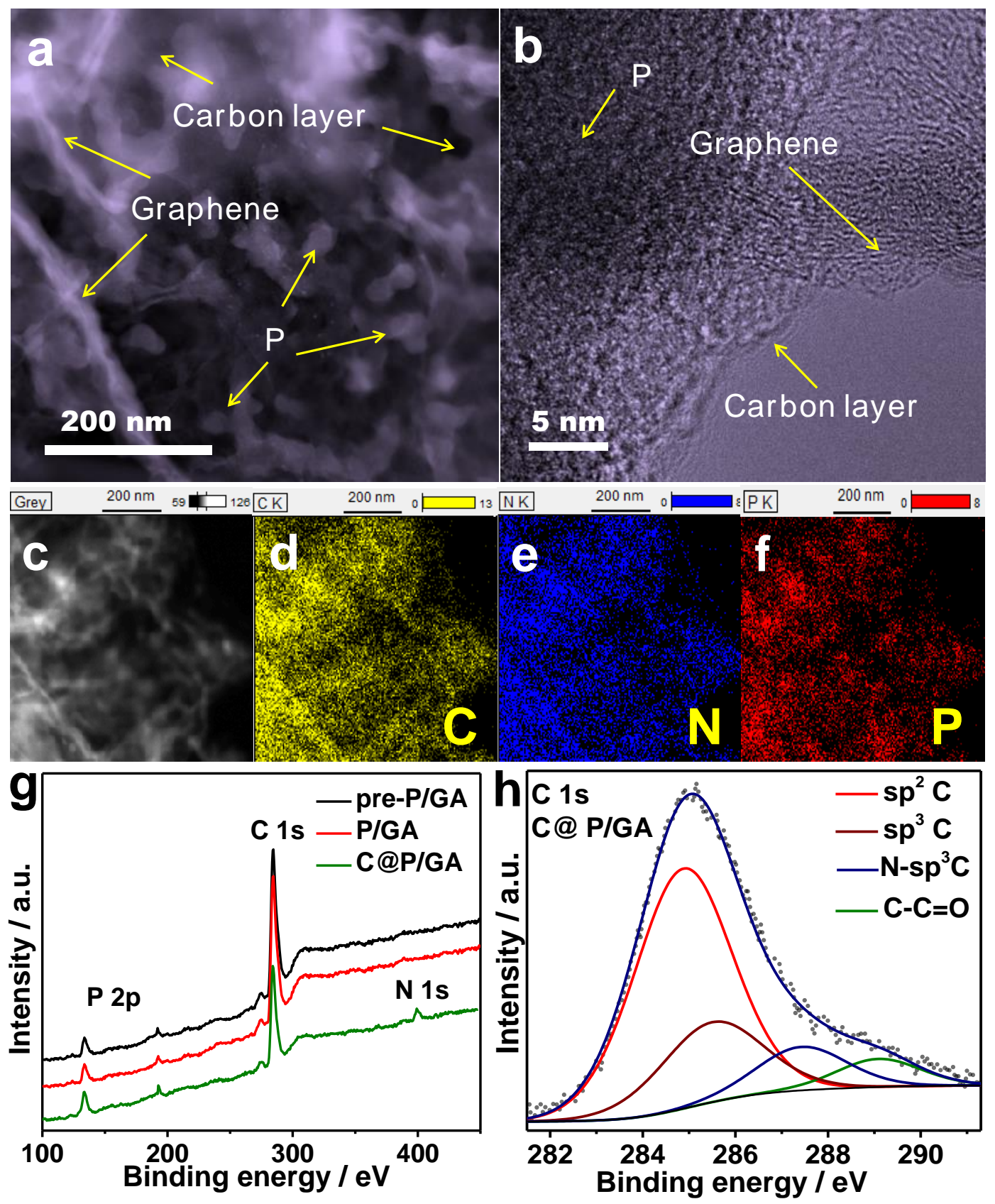

Figure 3. a) TEM and, b) HRTEM images, (c) STEM image and elemental mapping analysis of C@P/GA: (d) carbon, (e) nitrogen, (f) phosphorus. g, h) XPS analysis: g) survey spectra of pre-P/GA, P/GA, and C@P/GA composites, and h) fitted high-resolution C 1s XPS spectrum of C@P/GA composite. 

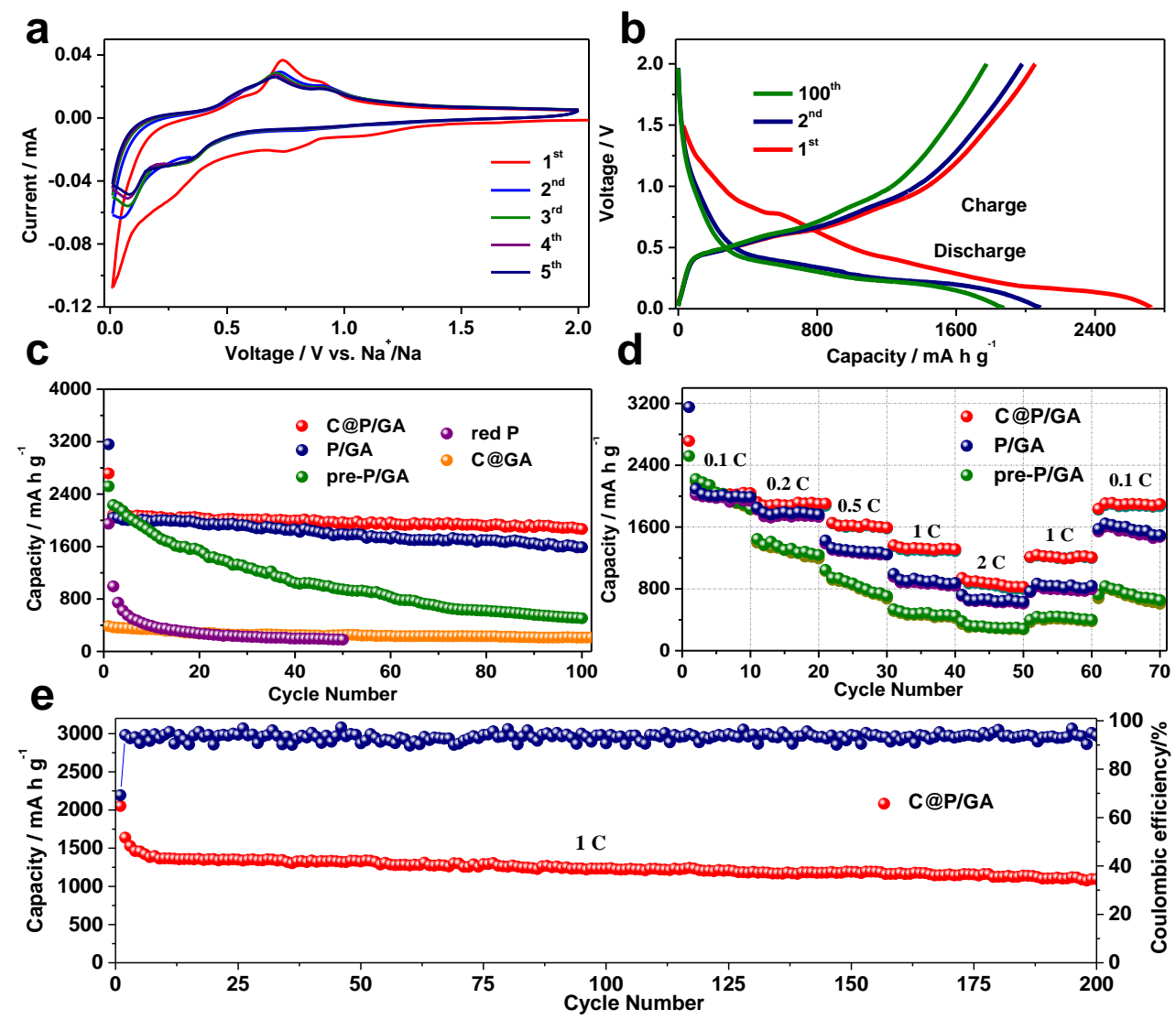

Figure 4. a) Cyclic voltammograms for the first 5 cycles of $C @ P / G A$ composite. b) Chargedischarge voltage profiles for selected cycles of C@P/GA composite at a current density of 0.1 C. c) Cycling performance of pre-P/GA, P/GA, C@P/GA, red P, and C@GA composites at 0.1 C. d) Rate performance of pre-P/GA, P/GA, and C@P/GA composites at different current densities. e) Cycling performance of $\mathrm{C} @ \mathrm{P} / \mathrm{GA}$ composite at $1 \mathrm{C} .\left(1 \mathrm{C}=2600 \mathrm{~mA} \cdot \mathrm{g}^{-1}\right)$. 


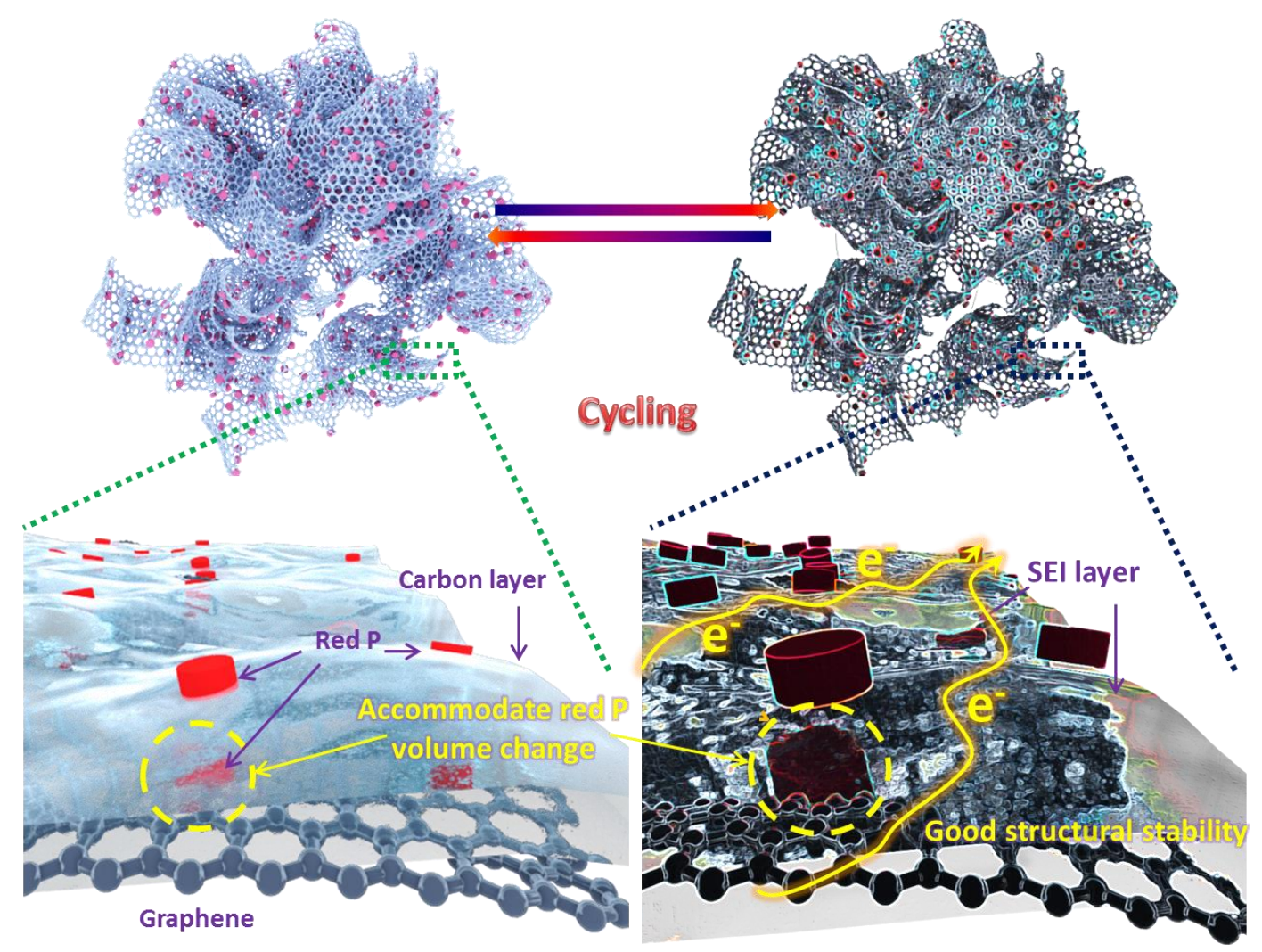

Scheme 2. Schematic illustration of the C@P/GA electrode in the SIB system. 
Integrated Carbon/red Phosphorus/graphene Aerogel Three-dimensional Architecture via Advanced Vapor-redistribution for High Energy Sodium-ion Batteries

Hong Gao, Tengfei Zhou, Yang Zheng, Yuqing Liu, Jun Chen *, Huakun Liu, Zaiping Guo *

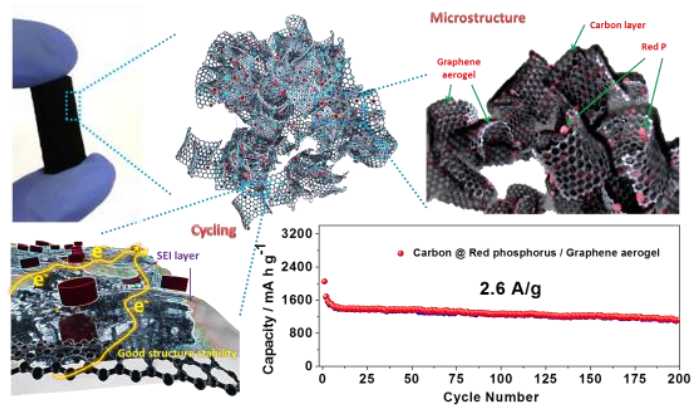

Three-dimensional hierarchical integrated carbon/red phosphorus/graphene aerogel composite is fabricated via an advanced vapor-redistribution technique. The red $\mathrm{P}$ nanoparticles (10-20nm) in the composite uniformly distribute between the graphene aerogel and carbon coating layer, which integrated into a 3D porous structure. The as-prepared C@P/GA electrode delivers a capacity of $1095.5 \mathrm{mAh} \cdot \mathrm{g}^{-1}$ after 200 cycles at $1 \mathrm{C}(1 \mathrm{C}=2600$ $\left.\mathrm{mA} \cdot \mathrm{g}^{-1}\right)$. 